\title{
Association between the use of gel pads under patients' knees and the incidence of peroneal neuropathy following liver transplantation
}

Gaab Soo $\underline{K i m}^{1}, M D$, Jin Sun $\underline{Y o o n}^{2}, M D, R i m \underline{K e e^{2}}$, MD, Young Hee $\underline{S h i n}^{1}$, MD, Justin Sangwook $\underline{K o}^{1}$, MD, Mi Sook Gwak ${ }^{1}$, MD, Ji Hye $\underline{H w a n g}^{3}$, MD, Suk-Koo $\underline{L e e}^{4}, M D$

INTRODUCTION There have been intermittent reports of peroneal neuropathy (PN) occurring after liver transplantation. Although PN may not be viewed as a serious complication by liver transplant (LT) recipients who require the transplant for survival, PN can significantly reduce quality of life. The incidence of PN appears to have increased after the use of gel pads was introduced. These gel pads, which are placed under patients' knees during surgery, are used to reduce lower back strain and prevent contact between the peroneal nerve at the fibular head and the hard surface of the operating table. The aim of the present study was to investigate the association, if any, between the use of gel pads and the incidence of PN. METHODS The medical records of 261 adult LT recipients were retrospectively reviewed. The recipients were divided into gel pad $(n=167)$ and non-gel pad $(n=94)$ groups. The incidence and possible risk factors of PN were compared between the two groups.

RESULTS The overall incidence of PN was $8.0 \%(21 / 261)$. The occurrence of PN was significantly higher in the gel pad group than in the non-gel pad group $(10.8 \%$ vs. $3.2 \%$; $<0.05)$. Other possible risk factors were comparable between the two patient groups.

CONCLUSION As the use of gel pads may increase the incidence of PN, we recommend against the use of gel pads under the knees of LT recipients.

Keywords: liver transplantation, patient positioning, peroneal neuropathy

\section{INTRODUCTION}

The incidence of peroneal neuropathy (PN) with foot drop has occasionally been reported after liver transplantations, with its incidence ranging from $1.4 \%$ to $13.3 \%$. $^{(1-5)}$ Although PN may not appear to be a serious complication in liver transplant (LT) recipients who require the transplant for survival, PN would greatly compromise the activity of the patient, resulting in extended hospital stay and decreased quality of life.

Postoperative neuropathies such as $\mathrm{PN}$ are mainly a consequence of nerve compression due to inadequate positioning of the nerve for a long period of time. ${ }^{(6)}$ In a previous study, we found that 33 out of 623 LT recipients developed PN over 12 years. ${ }^{(1)}$ While the perioperative use of intermittent pneumatic compression (IPC) devices for prophylaxis against deep vein thrombosis, together with the tilting of the operating table during transplantation, was initially thought to be the predisposing cause of $\mathrm{PN}$, we found that the occurrence of $\mathrm{PN}$ did not decrease even after discontinuing the use of IPC devices. Thus in the present study, we looked into the possibility that the increased incidence of PN was due to the application of gel pads (GPs) under the patients' knees. GPs are used to reduce lower back strain and prevent contact between the peroneal nerve at the fibular head and the hard surface of the operating table. We hypothesised that the compression of the peroneal nerve by the GP during lateral tilting of the operating table is a probable cause of PN. This study was performed to investigate the association between the use of GPs under patients' knees and the incidence of $\mathrm{PN}$ after liver transplantation.

\section{METHODS}

After approval for the present study was obtained from the institutional ethics committee, the medical records of 261 adult patients who underwent liver transplantation from December 2008 to December 2011 were retrospectively reviewed, focusing on patients' electromyography results, nursing records, anaesthetic records, and consult papers for neurology, rehabilitation medicine and orthopaedics. Paediatric patients (i.e. patients aged $<14$ years), patients who underwent additional operations, patients who had prior neurologic deficiencies and patients with retransplantation were excluded from the study.

During liver transplantation, patients are typically placed in the neutral position on the operating table, which is covered with a gel mat over a warming blanket. For surgical convenience, the left arm of the patient is typically tucked at the patient's side and the right arm positioned on the arm board at an angle $<90^{\circ}$. The patient's legs are then placed parallel to each other, with the knees supported using a GP (Fig. 1). The size of the GP (SK-27; Sung Kwang Meditech, Seoul, Korea) used measured $47 \mathrm{~cm}$ (length) $\times$ $10 \mathrm{~cm}$ (width) $\times 5 \mathrm{~cm}$ (height). GPs were used in our institution until November 2010. 

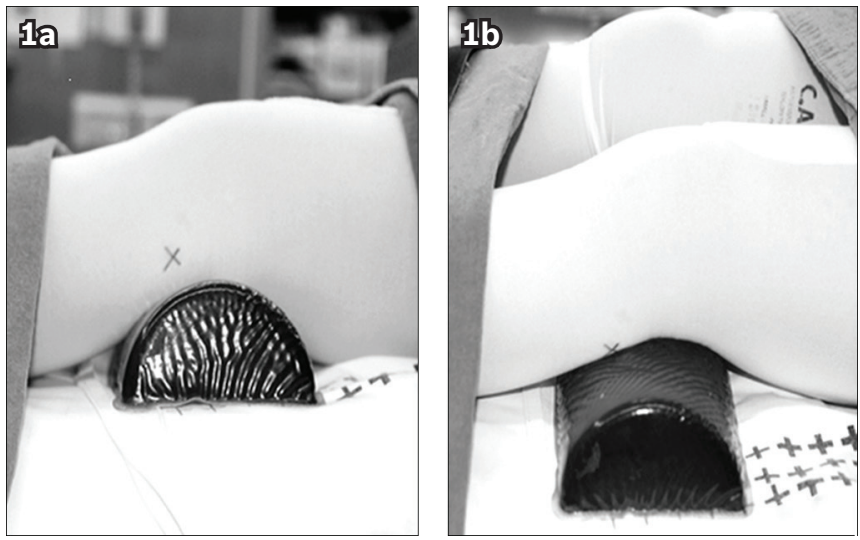

Fig. 1 Photographs show the position of the fibular head in relation to the gel pad, according to the patient's position on the operating table. (a) In the supine position, the fibular head is free. (b) However, in the laterally tilted position, the fibular head is in contact with the gel pad. Both photographs were taken at the same level.

The $\mathrm{LT}$ recipients in our study were assigned to either the GP $(n=167)$ or the non-GP $(n=94)$ group, according to whether the GP was applied. The incidence and possible risk factors of PN, such as age, gender, body mass index (BMI), diabetes mellitus, history of smoking, history of alcohol use, source of allograft, severity of liver disease (assessed using the model for end-stage liver disease [MELD] scoring system) and operation time, were compared between the two groups.

Data analysis was performed using the Statistical Package for the Social Sciences for Windows version 14.0 (SPSS Inc, Chicago, IL, USA). Data was expressed as either mean \pm standard deviation or number (percentage) of patients. Discrete variables were analysed using chi-square or Fisher's exact test. Either Student's $t$-test or Mann-Whitney $U$ test was used for the analysis of continuous variables. A p-value $<0.05$ was considered statistically significant.

\section{RESULTS}

In the present study, 18 LT recipients in the GP group $(n=167)$ and 3 in the non-GP group $(n=94)$ were diagnosed with PN after liver transplantation. The overall incidence of $\mathrm{PN}$ in our study cohort of 261 LT patients was $8.0 \%$. The incidence of PN was significantly higher in the GP group than in the non-GP group $(10.8 \%$ vs. $3.2 \%$; p < 0.05) (Table I). PN was not observed to be predominant in either leg. Other possible risk factors, such as age, gender, BMI, diabetes mellitus, history of smoking, history of alcohol use, source of allograft, MELD score and operation time, were comparable between the two groups.

\section{DISCUSSION}

During surgeries, GPs are used under the knees of patients to reduce lower back strain and prevent peroneal nerve compression at the fibular head due to contact with the hard surface of the operating table. However, the present study provides evidence that the use of GPs under the knees of patients may be a contributing factor for $\mathrm{PN}$. While adequate padding usually decreases the risk of postoperative neuropathy, the inappropriate use of paddings may actually cause postoperative neuropathy.
Table I. Characteristics of liver transplant recipients $(n=261)$.

\begin{tabular}{|c|c|c|c|}
\hline \multirow[t]{2}{*}{ Characteristic } & \multicolumn{2}{|c|}{ No. of patients (\%) } & \multirow[t]{2}{*}{ p-value } \\
\hline & $\begin{array}{l}\text { Gel pad } \\
\text { group } \\
(n=167)\end{array}$ & $\begin{array}{c}\text { Non-gel pad } \\
\text { group } \\
(n=94)\end{array}$ & \\
\hline Age* (yrs) $^{*}$ & $50.0 \pm 9.3$ & $51.6 \pm 8.4$ & 0.47 \\
\hline Gender & & & 0.19 \\
\hline Male & $121(72.5)$ & $75(79.8)$ & \\
\hline Female & $46(27.5)$ & $19(20.2)$ & \\
\hline $\mathrm{BMI}^{*}\left(\mathrm{~kg} / \mathrm{m}^{2}\right)$ & $24.3 \pm 4.1$ & $24.3 \pm 3.2$ & 0.51 \\
\hline Diabetes mellitus & $37(22.2)$ & $17(18.1)$ & 0.44 \\
\hline History of smoking & $30(18.0)$ & $19(20.2)$ & 0.84 \\
\hline History of alcohol use & $36(21.6)$ & $22(23.4)$ & 0.73 \\
\hline Source of allograft & & & 0.68 \\
\hline Living donor & $137(82.0)$ & $79(84.0)$ & \\
\hline Cadaver & $30(18.0)$ & $15(16.0)$ & \\
\hline MELD score* & $20.8 \pm 10.8$ & $19.0 \pm 10.9$ & 0.13 \\
\hline Operation time* (mins) & $672.8 \pm 113.9$ & $668.0 \pm 121.7$ & 0.17 \\
\hline PN & $18(10.8)$ & $3(3.2)$ & $<0.05$ \\
\hline Side of PN & & & 0.18 \\
\hline Right leg & $3(1.8)$ & $1(1.1)$ & \\
\hline Left leg & $6(3.6)$ & $1(1.1)$ & \\
\hline Both legs & $9(5.4)$ & $1(1.1)$ & \\
\hline
\end{tabular}

* Data is presented as mean \pm standard deviation. BMI: body mass index; MELD: model for end-stage liver disease; PN: peroneal neuropathy

The incidence of PN after liver transplantation was reported to be $1.4 \%-13.3 \%$ in previous studies, ${ }^{(1-5)}$ which is comparable to the overall incidence of PN in the present study (21/261, 8.0\%).

In a previous study, we investigated the effect of the use of IPC devices on the occurrence of PN in LT patients. ${ }^{(1)}$ We found that when IPC devices were not used perioperatively, the incidence of PN was $2.9 \%$. In that study, we did not use support devices under the patients' knees, and the patients were subject to similar surgical conditions (in terms of surgical position during the operation) as the patients from the non-GP group in the present study. The present study demonstrated that the incidence increased to $10.8 \%$ after the use of GPs was introduced in our institution. This incidence dropped to $3.2 \%$ after the use of GPs was ceased, strongly suggesting that the use of GPs plays a decisive role in the development of $\mathrm{PN}$.

In previous studies, it was found that PN developed more frequently in the left leg - a finding that was associated with prolonged intraoperative tilting of the operating table toward the left to facilitate mobilisation of the liver. ${ }^{(1,5)}$ However, in the present study, predominance of PN in the left leg was not observed, and as we did not measure the degree and duration of the tilting of the operating table, we were unable to determine whether these aspects differed from the previous study. ${ }^{(1)}$ The present study's result may be ascribed to the fact that surgeons in our institution are repeatedly reminded that excessive tilting of the operating table may result in an increased risk of PN, leading to the surgical team's avoidance of exaggerated tilting of the table.

Compression or stretching of the peroneal nerve due to the malposition of a patient is a well-known cause of $\mathrm{PN} .{ }^{(6-13)} \mathrm{PN}$ can develop if the peroneal nerve is compressed between the fibular head and external structures such as an operating table. In our 
institution, GPs were placed under the knees of LT recipients to reduce lower back strain and prevent contact between the peroneal nerve at the fibular head and a hard surface, according to the recommendation by the Association of periOperative Registered Nurses. ${ }^{(14)}$ Also, LT recipients were positioned in the neutral position during surgery, as recommended in Perioperative Standards and Recommended Practice. ${ }^{(15)}$ In order to minimise the risk of nerve injury, safety measures such as ensuring that there was adequate padding to protect the saphenous, sciatic and peroneal nerves were taken. However, firm conclusions regarding the most effective means of intraoperative pressure relief could not be drawn. ${ }^{(15)}$ One study insisted rolled sheets and towels not be used because they cause pressure. ${ }^{(16)}$

The peroneal nerve at the fibular head may have been compressed by the GP when the operating table was tilted laterally, and this might have increased the incidence of PN. The common peroneal nerve winds around the neck of the fibula, and this superficial segment of the common peroneal nerve is covered only by subcutaneous tissues and skin. This anatomic characteristic of the peroneal nerve makes it more vulnerable to compression injury. Moreover, frequent tilting of the operating table during surgery to facilitate liver mobilisation for surgical convenience may aggravate the condition..$^{(1,3,5)}$

Most incidences of postoperative $\mathrm{PN}$ are associated with surgeries performed in the lithotomy position; postoperative $\mathrm{PN}$ is uncommon when the supine position is used. ${ }^{(12,13)}$ In a retrospective review of LT recipients, Moreno et al found that 2 of $143(1.4 \%)$ adult LT recipients developed PN during the postoperative period due to the compression caused by knee supports that were placed in the popliteal space when patients were in the supine position. ${ }^{(2)}$ Moreno et al emphasised that supports placed in the popliteal fossa should not compress the head of the fibula since such compression results in lesions of the nerve corresponding to this level.(2) Careful positioning of $\mathrm{LT}$ recipients on the operating table and measures to protect the peroneal nerve are needed to prevent the occurrence of PN after liver transplantation.

In the present study, we did not observe any significant difference between the GP and non-GP groups with regard to the potential risk factors for $\mathrm{PN}$ (i.e. age, gender, BMI, diabetes mellitus, history of smoking, history of alcohol use, source of allograft, MELD score and operation time). The predisposing risk factors for PN in LT recipients have yet to be well established. In a study by Singhal et al, ${ }^{(5)}$ which investigated the predisposing risk factors for $\mathrm{PN}$ following liver transplantation, the following characteristics were found to be associated with $\mathrm{PN}$ : tall and slender body shape $\left(\mathrm{BMI}<20 \mathrm{~kg} / \mathrm{m}^{2}\right)$, a history of severe and rapid weight loss (> $15 \mathrm{~kg}$ in six months), and poor nutritional status $(35 \mathrm{mg} / \mathrm{dL}<$ serum cholesterol $<56 \mathrm{mg} / \mathrm{dL}$ ). Other studies have identified a number of other features as potential risk factors for lower extremity neuropathies in the perioperative period, and this includes thin body shape, ${ }^{(5)}$ a history of smoking, ${ }^{(17)}$ a history of alcohol use, ${ }^{(18)}$ presence of diabetes mellitus, ${ }^{(19)}$ anatomic anomalies, ${ }^{(20)}$ prolonged duration in lithotomy, ${ }^{(5)}$ and positioning beyond the comfortable range of motion. ${ }^{(19)}$
Patients who develop PN after liver transplantation are usually treated conservatively using physical therapy, with gradual improvement over time. ${ }^{(2,3)}$ In general, nerve injury caused by intraoperative malpositioning is treated symptomatically. ${ }^{(21)}$ Pain is treated with analgesics or other medications (e.g. gabapentin) that are effective in relieving neurologic pain. Motor deficit is treated using physical therapy that aims to maintain the flexibility of the joint, as well as improve the range of motion. The use of a splint may be beneficial in protecting the extremity from further injuries and assisting in daily life activities, and surgery is recommended when the lesions do not heal. The types of surgery that may be applicable are neurolysis, intraoperative evaluation of neuroma incontinuity, and transposition and grafting of nonconducting lesions. After surgical treatment, the patient's motor power generally returns to normal within six months. ${ }^{(2,3,5)}$

It is generally uncertain when PN develops following liver transplantation. This is because patients are only able to complain about neurologic symptoms after they recover consciousness, and it may take several days or even weeks for the symptoms of PN to surface. Also, as it is not uncommon for $\mathrm{LT}$ recipients to remain unconscious in the ICU for several days after surgery, the injury may have occurred during the patient's stay in the ICU. In other words, careful positioning of the patient during the ICU stay is as important as careful positioning of the patient during surgical procedures. The medical team should bear in mind the possibility of PN development during the care and treatment of patients.

In conclusion, the present study found that the use of GPs under the knees of patients was found to be associated with an increased incidence of $\mathrm{PN}$ after liver transplantation. The use of padding devices such as GPs, which are intended to reduce lower back strain and prevent contact between the peroneal nerve and hard surfaces, may actually increase the incidence of PN instead of reducing it. Therefore, we recommend against the use of GPs under the knees of LT recipients. Future studies on alternative measures for the prevention of PN are needed.

\section{REFERENCES}

1. Yoon JS, Gwak MS, Yang M, et al. Peroneal neuropathy after liver transplantation. Transplant Proc 2008; 40:2552-3.

2. Moreno E, Gómez SR, Gonzalez I, et al. Neurologic complications in liver transplantation. Acta Neurol Scand 1993; 87:25-31.

3. Nonthasoot B, Sirichindakul B, Nivatvongs S, Sangsubhan C. Common peroneal nerve palsy: an unexpected complication of liver surgery. Transplant Proc 2006; 38:1396-7.

4. Atalay A, Karatas M, Turhan N, et al. Bilateral drop-foot after orthotopic liver transplant. Transplant Proc 2006; 38:1471-3.

5. Singhal A, Varma M, Goyal N, et al. Peroneal neuropathy following liver transplantation: possible predisposing factors and outcome. Exp Clin Transplant 2009; 7:252-5.

6. Sawyer RJ, Richmond MN, Hickey JD, Jarrratt JA. Peripheral nerve injuries associated with anaesthesia. Anaesthesia 2000; 55:980-91.

7. Borgeat A, Ekatodramis G. Nerve injury associated with regional anesthesia. Curr Top Med Chem 2001; 1:199-203.

8. Britt BA, Gordon RA. Peripheral nerve injuries associated with anaesthesia. Can Anaesth Soc J 1964; 11:514-36.

9. Cheney FW, Domino KB, Caplan RA, Posner KL. Nerve injury associated with anesthesia: a closed claims analysis. Anesthesiology 1999; 90:1062-9.

10. Kroll DA, Caplan RA, Posner K, Ward RJ, Cheney FW. Nerve injury associated with anesthesia. Anesthesiology 1990; 73:202-7.

11. Sirinan C, Akavipat P, Srisawasdi S, Tanudsintum S, Weerawatganon T. The 
Thai Anesthesia Incidents Study (THAI Study) on nerve injury associated with anesthesia. J Med Assoc Thai 2005; 8888 Suppl 7:S102-5.

12. Warner MA, Martin JT, Schroeder DR, Offord KP, Chute CG. Lowerextremity motor neuropathy associated with surgery performed on patients in a lithotomy position. Anesthesiology 1994; 81:6-12.

13. Warner MA, Warner DO, Harper CM, Schroeder DR, Maxson PM. Lowe extremity neuropathies associated with lithotomy positions. Anesthesiology 2000; 93:938-42.

14. McEwen DR. Intraoperative positioning of surgical patients. AORN J 1996; 63:1059-63.

15. Recommended practices for positioning the patient in the perioperative setting. In: Conner R, Blanchard JC, Reno D, AORN, eds. Perioperative
Standards and Recommended Practices. Denver: AORN, 2009: 525-48.

16. ERCI. Pressure ulcers. Healthcare Risk ControlHRC Risk Analysis. 2006; 3(Nursing 4):1-37.

17. Walton-Geer PS. Prevention of pressure ulcers in the surgical patient. AORN J 2009; 89:538-48.

18. Parks BJ. Postoperative peripheral neuropathies. Surgery 1973; 74:348-57.

19. Dawson DM, Krarup C. Perioperative nerve lesions. Arch Neurol 1989; 46:1355-60

20. Romfh JH, Currier RD. Sciatic neuropathy induced by the lithotomy position. Arch Neurol 1983; 40:127.

21. Winfree CJ, Kline DG. Intraoperative positioning nerve injuries. Surg Neurol 2005; 63:5-18 